INRA Prod. Anim., 2017, 30 (4), 395-406

\title{
Des services contrastés rendus par l'élevage dans les espaces urbains et périurbains européens
}

\author{
C. DELFOSSE', B. DUMONT', N. HOSTIOU
}

${ }^{1}$ Université de Lyon, Laboratoire d'Etudes Rurales, 69363, Lyon, France 2 Université Clermont Auvergne, INRA, VetAgro Sup, UMR Herbivores, 63122, Saint-Genès-Champanelle, France ${ }_{3}^{3}$ Université Clermont Auvergne, INRA, AgroParisTech, Irstea, VetAgro Sup, UMR Territoires, 63000, Clermont-Ferrand, France

Courriel : bertrand.dumont@inra.fr

\begin{abstract}
Dans les villes européennes et à leur périphérie, l'élevage répond aux attentes des citoyens en matière d'alimentation (circuits courts...) et de qualité de vie (besoin de « nature »...). Nous analysons ici les services, pour certains spécifiques, rendus par l'élevage dans ces espaces, ainsi que les opportunités et les freins à son développement.
\end{abstract}

Cet article aborde la question du rôle, des impacts et des services rendus par l'élevage dans des territoires qui se caractérisent par de très fortes densités humaines et semblent de ce fait non voués à l'élevage, du moins en Europe. L'élevage urbain et périurbain fait pourtant l'objet d'un intérêt croissant de la part des consommateurs, dont une frange croissante est soucieuse de la provenance des aliments qu'ils consomment (BaysseLainé et Perrin 2017, Perrin et Soulard 2017). Cet intérêt se prolonge dans les réflexions sur les systèmes alimentaires locaux et territorialisés (Lardon et Loudiyi 2014), et par l'action des collectivités territoriales relative à l'approvisionnement alimentaire des villes et à l'aménagement des espaces qui les entourent. D'un point de vue environnemental, rapprocher les centres de production des centres de consommation réduit les consommations d'énergie non renouvelables. Des systèmes d'élevage innovants à proximité immédiate des villes pourraient contribuer de façon notable à la production alimentaire en n'utilisant que peu de surfaces, d'eau et d'énergie ; c'est par exemple le cas des démarches d'écologie industrielles qui se fondent pour une large part sur la valorisation et le recyclage des déchets (Dumont et al 2013, zu Ermgassen et al 2016). Dans ces espaces, les services rendus par l'élevage peuvent être très spécifiques (fermes pédagogiques...), tout comme les contraintes qui freinent son développement (coût du foncier, nuisances pour les citadins).

En Europe, l'élevage urbain et périurbain revêt des formes très diverses qui comprennent des formes professionnelles « classiques », mais aussi des élevages destinés à approvisionner les circuits courts, à l'autoconsommation, des élevages amateurs de loisir ou à visée pédagogique. Notre objectif est ici de synthétiser cette littérature souvent fragmentaire, et de structurer cette analyse grâce au cadre des bouquets de services, qui permet de considérer la coexistence d'effets positifs et négatifs d'une activité (ici l'activité d'élevage) à un instant donné, dans un territoire (Raudsepp-Hearne et al 2010, Dumont et al 2017, ce numéro). Nous utilisons comme mode de représentation la « grange », élaborée à l'occasion de l'expertise scientifique sur les rôles, impacts et services issus des élevages en Europe (Dumont et al 2016) et détaillée par Duru et al (2017, ce numéro); celle-ci permet de représenter les interrelations entre services, et les pressions propres ou extérieures à chaque territoire d'élevage qui s'exercent sur celui-ci. Dans ces espaces à forte densité humaine, l'analyse des services rendus par l'élevage nécessite de distinguer les espaces urbains (ville centre et banlieue là où il y a peu d'espaces ouverts et une quasi absence de l'agriculture), des espaces périurbains, déstructurés par l'avancée de la ville, et où se jouxtent des zones fortement urbanisées et des zones où l'agriculture reste très présente. Nous montrerons en effet que les bouquets de services rendus par l'élevage dans ces territoires, ses atouts et les freins à son développement y sont très différents.

Dans une première partie de l'article, nous définissons ces deux types d'espaces et présentons les sources mobilisées pour analyser les services qu'y rend l'élevage dans chacun d'eux. Dans les deux parties suivantes, nous analysons les services d'approvisionnement, les services environnementaux et les services sociaux et de vitalité territoriale rendus par l'élevage dans les espaces urbains et périurbains, ainsi que les freins à son développement. Nous concluons en proposant quelques pistes de recherches relatives à la quantification du service d'approvisionnement, à la valorisation des déchets alimentaires, aux emplois créés et à la précarité de l'élevage dans ces espaces.

\section{1 / Définitions et sources mobilisées}

Nous retenons la définition suivante de l'agriculture urbaine : la culture de produits végétaux et animaux à l'intérieur même des villes (Lovell 2010), bien que certains chercheurs utilisent le terme d'agriculture urbaine de façon plus large (Ba et Aubry 2011, Nahmias et Le Caro 2012, Mundler et al 2014) qui intègre ce que nous considérons relever de l'agriculture périurbaine. Suivant notre définition, l'espace urbain apparaît comme assez homogène, malgré les différences de taille entre les métropoles et les villes moyennes. L'espace périurbain recouvre des réalités plus variées (Arnal 2012). Les systèmes d'élevage contribuent aux schémas de gouvernance alimentaire locale qui visent à mieux relier les villes à leur agriculture de proximité (Perrin et Soulard 2014). Cependant, la situation 
de l'élevage diffère entre les fronts de périurbanisation et les espaces périurbains " stabilisés ». En particulier, là où le phénomène de périurbanisation est ancien, les élus et les habitants tendent à se revendiquer non plus comme des espaces en instance d'urbanisation, mais comme des espaces « mi ville/mi campagne » (Vanier 2011), dans lesquels l'usage agricole reste important.

Cet article a été conduit à partir d'une revue de la littérature, dont nous présentons ici les principales sources mobilisées. La littérature scientifique est relativement abondante sur l'agriculture périurbaine en Europe, notamment en France, où les travaux développés depuis plusieurs années ont été réactivés par les recherches sur l' "Urban Food Planning » autour des enjeux d'aménagement pour répondre à la sécurité alimentaire des villes (Morgan 2009, Lardon et Loudiyi 2014). Ces travaux ont été initiés dans les métropoles d'Amérique du Nord et d'Europe du Nord au début des années 2000 et ont depuis gagné l'ensemble du continent européen (Zasada 2011, Bonnefoy et Brand 2014).

Les villes moyennes, également marquées par la périurbanisation, commencent à faire l'objet de recherches (Duvernoy 2002, Perrin et Soulard 2014), de quelques thèses (Lescureux 2003, Arnal 2012, Nougarèdes 2013) et de travaux de Master récents (Le Gall 2013, Tredan 2016) qui ont aussi été mobilisés dans cet article. Ces travaux s'intéressent aux liens entre la ville et l'agriculture périurbaine dans une perspective historique (Chatzimpiros et Barles 2010) et traitent du rôle culturel de l'élevage, et du rôle de la ville dans la valorisation de l'agriculture environnante. D'autres évoquent les liens entre le rayonnement métropolitain et la gastronomie qui lui est associée, et les modes de valorisation des produits de l'élevage dans les stratégies métropolitaines (Csergo et Lemasson 2008, Delfosse 2015). Enfin, il existe différents travaux (y compris des décisions de justice) sur les contentieux et les conflits en milieu périurbains (Henderson 2003, Torre et al 2015).

L'élevage en ville est bien moins étudié et n'a fait l'objet que de quelques publications depuis les années 2000 . Les travaux sur l'agriculture urbaine sont en effet principalement dévolus au maraichage et aux jardins. Les animaux de rente y sont rarement évoqués à l'exception de la volaille, des moutons et des chevaux (Blanc 2000, Darribehaude et al 2016). Quelques articles récents sur l'évolution des métropoles des Balkans apportent un éclairage complémentaire sur la place de l'élevage en ville (Guest 2003, Tribol 2017). Au vue de la rareté de la littérature scientifique, nous avons complété notre recherche bibliographique par quelques travaux de recherche hors Europe, mais relatifs à des situations analogues à celle des villes européennes (Voigt 2011, McClintock et al 2014, Ward et al 2014, Huang et Drescher 2015). Nous avons aussi consulté les sites internet de communautés urbaines et des sites associatifs traitant de l'alimentation, de la nature en ville ou même du jardinage.

\section{2 / Rôles, impacts et services rendus par l'élevage urbain}

L'élevage était une activité importante dans les villes européennes au XIXème siècle notamment pour l'approvisionnement en lait et en œufs des populations urbaines (Delfosse 2014). De nombreux animaux de trait y vivaient avant le développement de l'automobile (McShane et Tarr 2007, Baratay 2011). La présence d'abattoirs et de très importants marchés de gros pour la viande, comme celui de la Villette à Paris, entrainait la présence ponctuelle de nombreux animaux (Chemla 1994). Le développement de la chaîne du froid, des transports et l'évolution du traitement des produits laitiers (Delfosse 2014) et carnés ont éloigné les élevages de la ville. Le refus des nuisances et les règlements sanitaires et environnementaux ont participé à ce recul.

\section{1 / Un service d'approvision- nement contrasté entre les villes d'Europe de l'Ouest et de l'Est}

C'est essentiellement au travers des élevages de volailles de fond de cour, et plus récemment de volailles associées aux jardins partagés, que l'élevage contribue au service d'approvisionnement dans les villes d'Europe de l'Ouest. Les poules consomment les déchets ménagers et fournissent des œufs frais aux citadins (photo 1). Des municipalités et des intercommunalités urbaines organisent cet élevage en distribuant une à deux poules par ménage. Certains projets d'aviculture urbaine très structurés se sont ainsi développés à Laval ou à MontereauFault-Yonne en Ile-de-France. La consultation des sites internet des communautés urbaines et de sites associatifs montre que ce phénomène se développe en France. La nourriture est souvent directement consommée par celui qui la produit, ce qui dans le cas des ménages pauvres accroît la consommation de produits frais et donc la qualité de leur alimentation (Voigt 2011). Une étude australienne a permis de quantifier que le maraîchage et l'aviculture de fond de cour (œufs et poulets de chair) pouvait couvrir entre 10 et $15 \%$ des besoins protéiques de ceux qui les mettent en œuvre (Ward et al 2014).

De manière plus marginale, les ruchers se développent à nouveau dans les parcs urbains, sur les toits (à Paris sur ceux du Grand Palais et de Bercy), et des mesures ont été prises dans certaines villes européennes pour permettre leur exploitation. L'aquaponie participe au recyclage des déchets de l'aquaculture par un compartiment végétal de type hydroponique (maraichage, culture ornementale) connecté à un bassin d'élevage en circuit fermé (Klinger et Naylor 2012, Love et al 2015). Elle pourrait réinscrire l'élevage et la commercialisation de

Photo 1. Affiche posée sur le portail d'une maison d'un ancien quartier ouvrier d'Amiens (Photographie Adrien Baysse-Lainé).

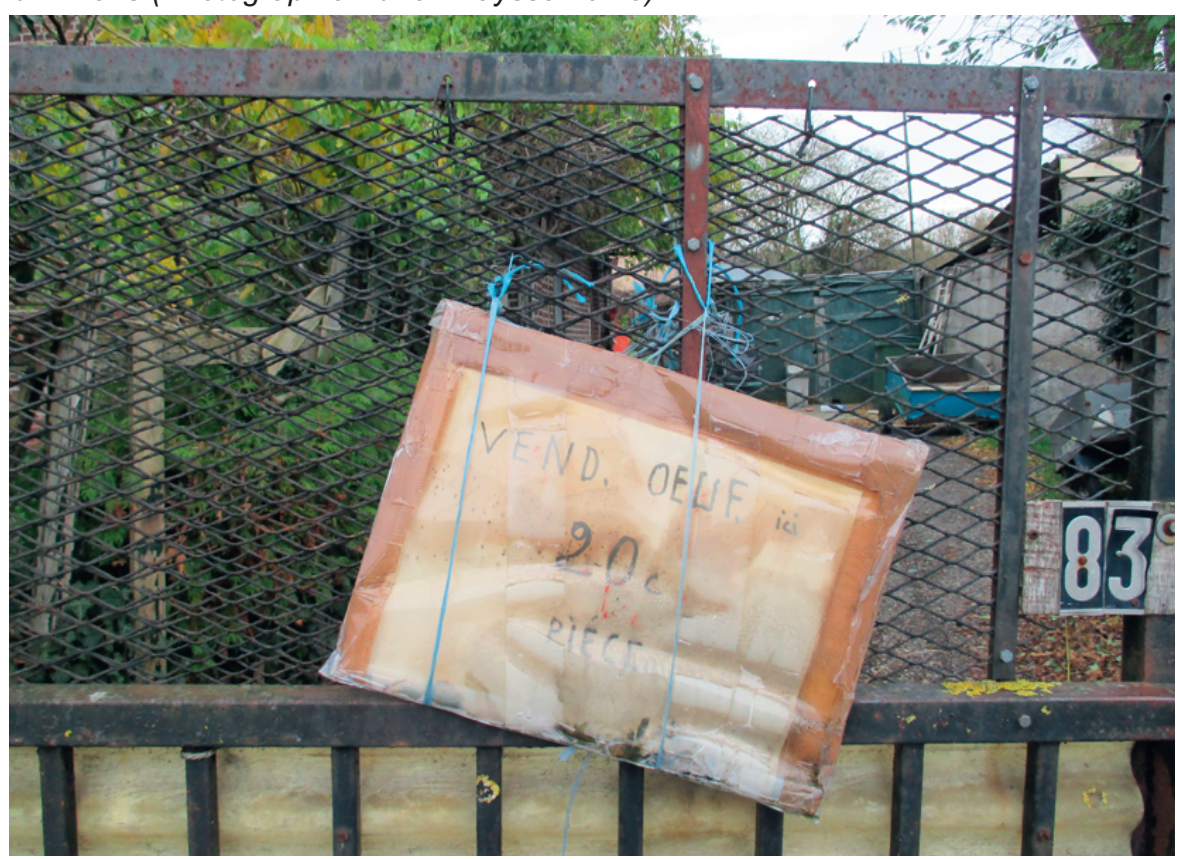


Photos 2 et 3. Brebis de race Thones et Marinthod pâturant sur le campus de Nanterre avec un berger (gauche); Moutons écossais autour du plan d'eau de la base de loisirs de Bourg-en-Bresse (droite).

On peut lire dans la revue d'information de la ville de Bourg-en-Bresse de mai-juin 2017 (C'est à Bourg, n²48) : « Fin avril, $13000 \mathrm{~m}^{2}$ seront mis en éco-pâturage dans la pente longeant le terrain de golf et sur les îles du parc de loisirs. Dans ces espaces clos, deux moutons de Soay originaires d'Ecosse et deux moutons d'Ouessant se chargeront de l'entretien de la pelouse. Une initiative respectueuse de la biodiversité, peu coûteuse et qui devrait être apprécié par les promeneurs ».
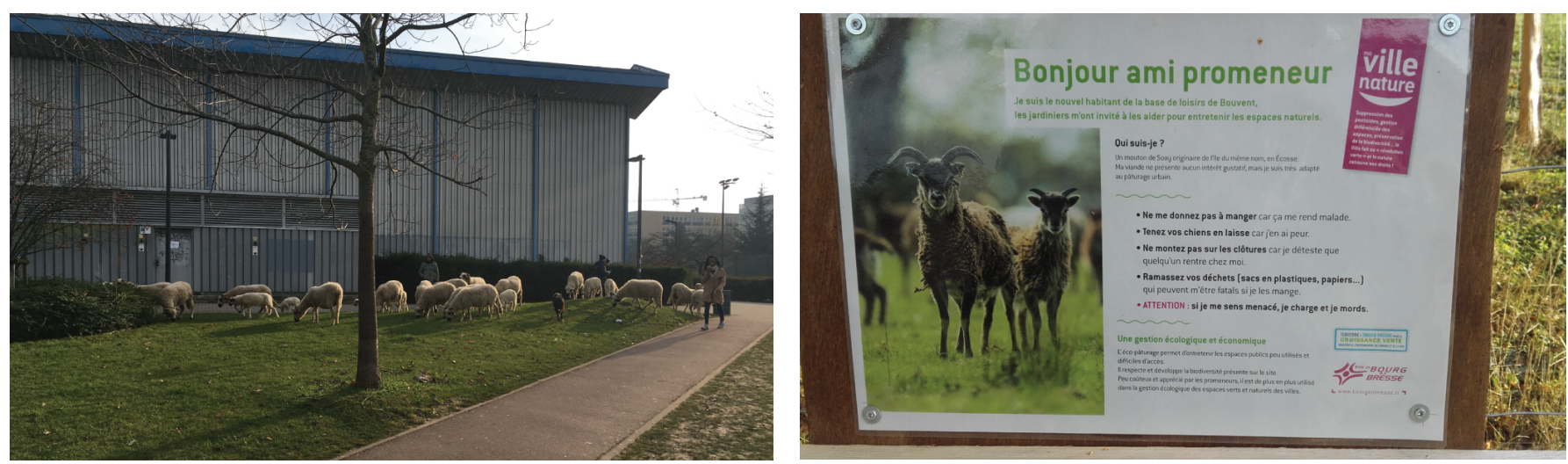

poissons dans des formes de maraichage. De même, les systèmes aquacoles en eau re-circulée nécessitent peu d'eau et de surfaces (Dumont et al 2013), et pourraient se développer dans les banlieues ou à la périphérie immédiate des villes puisqu'ils ne génèrent que peu de nuisances. Un des freins à leur développement est l'important investissement initial, mais celui-ci pourrait être rapidement rentabilisé par une commercialisation en vente directe.

L'importance du service d'approvisionnement est quantitativement plus importante dans les villes d'Europe de l'Est. Dans les Balkans, les formes d'urbanisation ont créé des espaces « vides » au sein de la trame urbaine qui ont été progressivement investis par différentes formes de pastoralisme (Guest 2003, Tribol 2017). Cependant les politiques visant à donner un statut de métropole moderne à ces villes provoquent un recul de ces espaces et donc du pastoralisme (Guest 2003). En Pologne et en Tchéquie (Smith et Jehlicka 2013), l'imbrication culturelle entre le monde rural et urbain permet le maintien d'élevages domestiques au sein d'un tissu urbain dense. Les crises économiques semblent par ailleurs réhabiliter l'autoconsommation ainsi que l'élevage domestique, notamment en Europe de l'Est, méditerranéenne et dans les Balkans (Jouve et Padilla 2007, Vallerand et al 2007, Perucho et al 2015). Par exemple, les lapins et les cochons sont fortement intégrés aux systèmes d'autoconsommation des villes d'Europe de l'Est, tout en participant à la réduction des déchets ménagers.

\section{2 / L'élevage en ville rend de nombreux services environne- mentaux et paysagers}

L'introduction d'animaux d'élevage dans les villes d'Europe de l'Ouest répond pour beaucoup au besoin de « nature en ville » des citadins. Celui-ci s'est d'abord traduit par la promotion des espaces verts, puis par l'introduction du maraichage dans des espaces communs. L'aviculture participe aussi à la réflexion sur la place des jardins dans les « smart cities » et contribue à la réduction des déchets alimentaires. Les petits ruminants sont principalement utilisés pour gérer de manière " écologique " les pelouses et les espaces interstitiels en réduisant les pollutions liées aux produits chimiques et aux hydrocarbures (Bories et Eychenne 2016). Le maintien de ces espaces naturalisés pourrait avoir une importance accrue en limitant les « ilots de chaleurs » que constituent les villes lors des canicules (Milan et Creutzig 2015). Lorsque l'élevage est introduit dans des espaces interstitiels précédemment abandonnés (comme par exemple sur les coteaux de Besançon), ce " reverdissement " procure un bénéfice esthétique aux habitants du voisinage qui retrouvent un usage récréatif à ces espaces. Les métropoles mènent des politiques actives de reconquête de ces espaces interstitiels pour y installer des exploitations agricoles. A Nantes, les installations agricoles ont en priorité concerné le maraîchage, mais les exploitations d'élevage arrivent en deuxième position (Barreau 2014).

La réintroduction par les municipalités de petits ruminants pour gérer les espaces verts ou les friches est encouragée par la législation sur le non-recours aux pesticides en ville (Darly 2014). Les animaux sont gérés directement par la municipalité, par des associations spécifiques ou par des entreprises d'entretien d'espaces verts. Ils sont conduits par un berger qui les amènent de parcelle en parcelle éparpillées dans le tissu urbain, et dans de rares cas ils pâturent seuls. Ces actions s'accompagnent souvent d'une volonté de valoriser la biodiversité domestique en utilisant des animaux de races à petits effectifs. C'est par exemple le cas à Bourg-en-Bresse (photos 2 et 3 ) et à Grand Synthe, commune de la banlieue de Dunkerque, qui a conclu un partenariat avec le centre régional de ressources génétique du Nord-Pas-deCalais pour gérer ces espaces interstitiels avec des animaux de races régionales.

\section{3 / Les rôles sociaux de l'élevage urbain}

Les nouvelles formes que prend l'élevage en ville (notamment l'entretien des espaces verts) créent quelques emplois, souvent pour réinsérer des personnes en difficulté (Voigt 2011, Darly 2014). La réintroduction des animaux dans le tissu urbain est largement motivée par une volonté de créer du lien social et constitue un facteur d'animation, d'éducation du public et d'insertion sociale et professionnelle (Etard 2014, Schneider 2014). Par exemple les chevaux et les ânes bâtés en ville, qui remplacent les véhicules motorisés pour le ramassage des ordures, le transport de personnes et des missions de surveillance, ont également un rôle social car ils permettent d'engager le dialogue entre habitants et employés municipaux (Vial et al 2015). Les fermes dites d'animation urbaine jouent aussi ce rôle (Darribehaude et al 2016) qu'elles soient temporaires ou plus pérennes. Parfois gérées par des associations, elles aident à rétablir un lien entre les citadins et l'élevage, en instruisant les citadins, qui pour beaucoup n'ont pas de racines rurales, sur la manière dont est produite la nourriture qu'ils consomment. Il est également à noter la présence de fermes thérapeutiques dans des hôpitaux (Darribehaude et al 2016) et de quelques centres équestres insérés dans l'espace urbain.

Enfin, l'élevage urbain a une fonction culturelle importante grâce aux fêtes autour des races locales, et aux produits 
Figure 1. Représentation synthétique du bouquet de service issu de l'élevage urbain.

Comme décrit par Duru et al (2017), le pentagone central représente un territoire et ses principales caractéristiques (les pictogrammes sont explicités ci-dessous). Les zones en vert symbolisent les prairies, en jaune les cultures et en gris les espaces artificialisés. Au sein du territoire, l'élevage interagit avec cinq interfaces. La nature et l'ampleur des effets sont représentés par une flèche sortante plus ou moins large et dont la couleur indique que ceux-ci sont positifs (vert), négatifs (rouge) ou mitigés (hachures); dans ce cas l'effet dominant borde la flèche. Sur l'interface travail et emploi, nous distinguons les emplois directs des emplois indirects (IAA..., flèche vers le personnage blanc). Les flèches entrantes indiquent l'utilisation d'intrants exogènes, des opportunités liées aux marchés (circuits courts) ou que l'élevage bénéficie de services intrants (flèche verte sur l'interface environnement et climat).

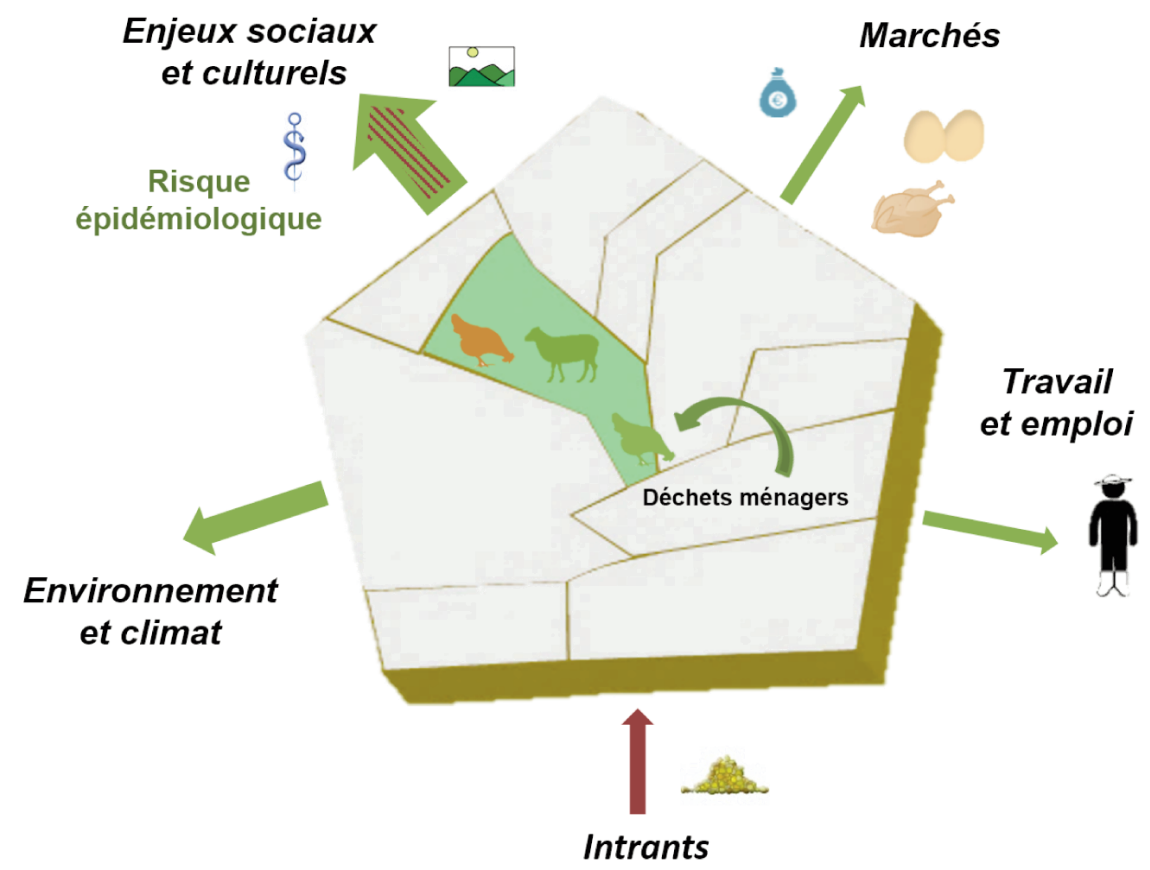

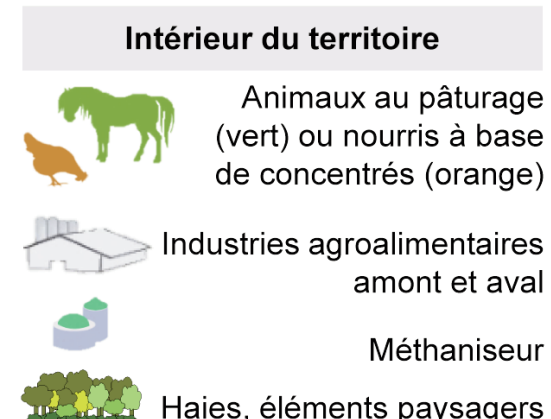

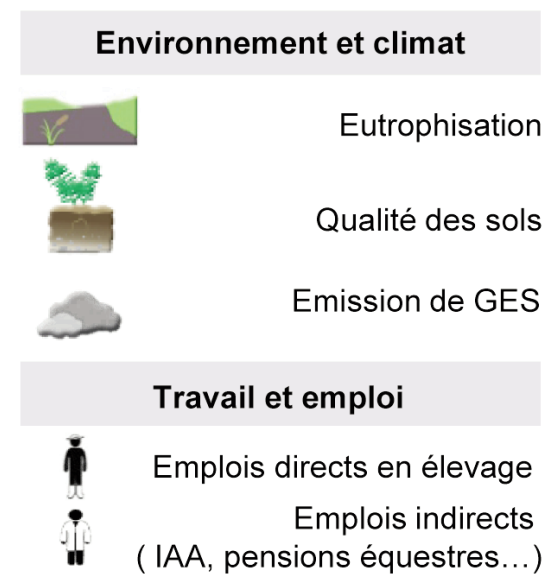

liés à l'élevage (Delfosse 2011, Arnal 2012), voire à la mise en scène des anciennes transhumances comme à Madrid (Labouesse 1998). La tauromachie, les courses taurines et les fêtes qui y sont
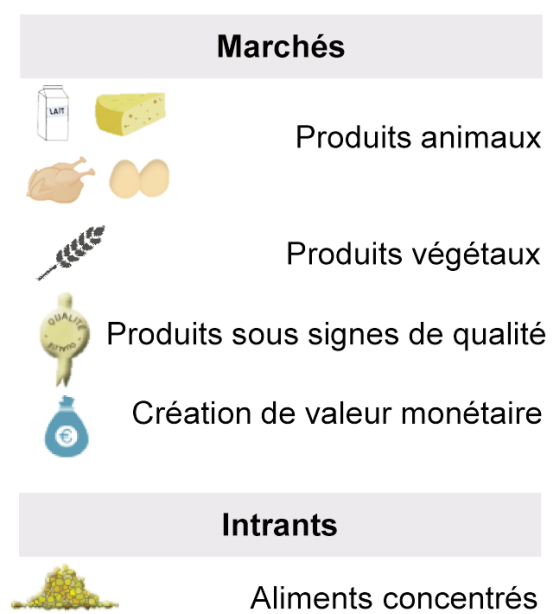

Engrais, pesticides, énergie

\section{Enjeux sociaux et culturels}

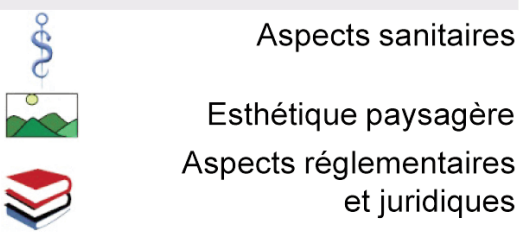

liées représentent des enjeux économiques importants (Maudet 2006). Les villes cherchent par ailleurs à mieux valoriser leur gastronomie en mettant à l'honneur les produits de l'élevage régio- nal (Delfosse 2015). La mise en scène patrimoniale, voire culturelle des produits et de l'élevage contribue alors à la construction identitaire, à l'animation et au rayonnement culturel de la ville. Elle 
Tableau 1. Atouts et impacts associés à l'élevage en ville.

\begin{tabular}{|c|c|c|}
\hline $\begin{array}{l}\text { Interfaces } \\
\text { étudiées }\end{array}$ & Effets positifs & Effets négatifs \\
\hline Intrants & $\begin{array}{l}\text { - Valorisation des déchets ménagers } \\
\text { - Economies d'énergie du fait des distances } \\
\text { réduites }\end{array}$ & $\begin{array}{l}\text { - Effets délocalisés pour la production } \\
\text { d'aliments concentrés }\end{array}$ \\
\hline Travail et emploi & $\begin{array}{l}\text { - Marginal en quantité mais intérêt pour } \\
\text { la réinsertion }\end{array}$ & \\
\hline Marchés & $\begin{array}{l}\text { - Approvisionnement en produits frais (intérêt } \\
\text { accru par la crise économique) }\end{array}$ & \\
\hline $\begin{array}{l}\text { Enjeux sociaux } \\
\text { et culturels }\end{array}$ & $\begin{array}{l}\text { - Lien social (cheval, jardins collectifs), } \\
\text { réinsertion } \\
\text { - Compréhension de la provenance de la } \\
\text { nourriture } \\
\text { - Usage récréatif (colombophilie) }\end{array}$ & $\begin{array}{l}\text { - Nuisances olfactives (déjection) et sonores } \\
\text { - Risque épidémiologique si contact étroit } \\
\text { avec l'homme (Influenza) } \\
\text { - Rapport à la mort de l'animal }\end{array}$ \\
\hline $\begin{array}{l}\text { Environnement } \\
\text { et climat }\end{array}$ & $\begin{array}{l}\text { - Moindre recours à la mécanisation et aux } \\
\text { pesticides pour entretenir espaces } \\
\text { interstitiels, qui contribuent à réduire les îlots } \\
\text { de chaleur }\end{array}$ & \\
\hline
\end{tabular}

peut aussi resserrer les liens ville-campagne (Delfosse 2011). Enfin, la colombophilie est encore très vivante dans les villes ouvrières d'Europe de l'Ouest (métropole lilloise, bassin minier du Nord de la France), comme de l'Est (villes minières de Silésie en Pologne, Delfosse 2001).

\section{4 / Des freins principalement liés aux nuisances et aux risques épidémiologiques occasionnés par les animaux}

La fourniture de ces services suppose un minimum de compromis dans les espaces à forte densité humaine, où la population urbaine a le plus souvent perdu toute familiarité avec les animaux d'élevage. Ceux-ci résultent en premier lieu des nuisances occasionnées par les animaux : odeurs liées aux déjections et à l'alimentation, et bruits tels que les aboiements de chiens de berger à Bucarest (Tribol 2017). L'élevage urbain accroît les risques épidémiologiques lorsque les animaux sont en contact étroit avec l'Homme. Les virus de type Influenza peuvent par exemple passer la barrière des espèces et atteindre l'homme soit par contact direct avec les volailles, soit par le biais du porc domestique qui en est un hôte intermédiaire. Leur virulence accrue après recombinaison pose alors un vrai problème de santé publique (Kuiken et al 2011).

Les débats relatifs à la législation autour de l'élevage en ville portent donc sur la réglementation de la vente pour les élevages domestiques, mais aussi sur les risques sanitaires encourus par les populations, et les nuisances olfactives et sonores. Aux États-Unis, il existe des réglementations qui limitent (voire inter- disent) l'élevage dans les zones urbaines (Voigt 2011, McClintock et al 2014). Au Canada, certaines municipalités font en revanche évoluer leur législation face à l'engouement pour l'élevage de fond de cour (Huang et Drescher 2015). Les compromis concernent également le foncier pour permettre le maintien de quelques espaces ouverts en ville. Les fermes urbaines à caractère associatif qui ne disposent pas de foncier et reposent sur des dispositifs alternatifs sont souvent dans des situations précaires, parfois à la limite de la légalité (Darly 2014). C'est par exemple le cas des élevages transhumants de Bucarest (Tribol 2017). La réintroduction de certaines formes d'élevage en ville semble plus soutenue dans les villes d'Europe de l'Ouest que dans celles de l'Est. Certains services rendus par les animaux d'élevage pourraient toutefois être remis en cause au titre du bien-être animal, en raison du stress des animaux des fermes itinérantes et de ceux introduits dans les hôpitaux pour accompagner les thérapies (Darribehaude et al 2016).

\section{5 / Un bouquet de services privi- légiant les services environnemen- taux et sociaux}

Les principaux impacts positifs ou négatifs de l'élevage en ville sont synthétisés dans la figure 1 et le tableau 1 en adoptant un formalisme inspiré d'une analyse socio-écologique (Duru et al 2017). La contribution des animaux d'élevage au service d'approvisionnement diffère entre les villes d'Europe de l'Ouest et de l'Est, sans que la littérature scientifique ne permette à ce jour de quantifier son importance. Les services environnementaux concernent l'entretien des espaces verts et dans une moindre mesure la réduction des déchets alimentaires. Enfin, la réintroduction des animaux en ville est souvent motivée par une volonté de créer du lien social et constitue un facteur de réinsertion et d'éducation des citadins vis-à-vis de l'origine des produits qu'ils consomment.

\section{3 / Rôles, impacts et services rendus par l'élevage dans les espaces périurbains}

L'élevage périurbain est présent dans les différents types de territoires européens cartographiés par Hercule et al (2017, ce numéro), mais avec des niveaux de présence différents. D'après les données du RGA de 2000, les grandes cultures sont en effet surreprésentées dans les couronnes périurbaines françaises, alors que l'élevage ovin et caprin, y est plus rare qu'ailleurs (Soulard et Thareau 2009). A l'inverse, les équidés y sont fortement représentés. La principale tendance est que la ville repousse l'élevage vers des espaces à plus faible densité humaine. Dans son processus d'étalement, la ville empiète sur des espaces ruraux qui étaient jadis voués à l'agriculture (photo 4). Les élevages hors-sol qui se sont développés dans les années 1970 dans les espaces périurbains ont tendance à s'en éloigner en raison des nuisances occasionnées par les animaux (Henderson 2003, Jouve et Padilla 2007). Cependant, certaines formes d'élevage bénéficient de la volonté d'une frange croissante de la population de recréer un lien direct avec ceux qui produisent la nourriture qu'ils consomment, par exemple grâce aux AMAP et aux drivefermiers. La précarité et les crises économiques peuvent aussi redynamiser de 
Photo 4. L'urbanisation rencontre des zones d'élevage dans l'espace périurbain de Montbrison, petite ville, elle-même relais de la périurbanisation stéphanoise (à gauche, photo $\mathrm{C}$. Delfosse) ; une ferme alternative dans le périurbain de Rome réintroduisant des races locales et cherchant à relocaliser le pecorino romano (fromage de Rome) essentiellement produit en Sardaigne (à droite, photo C. Delfosse).

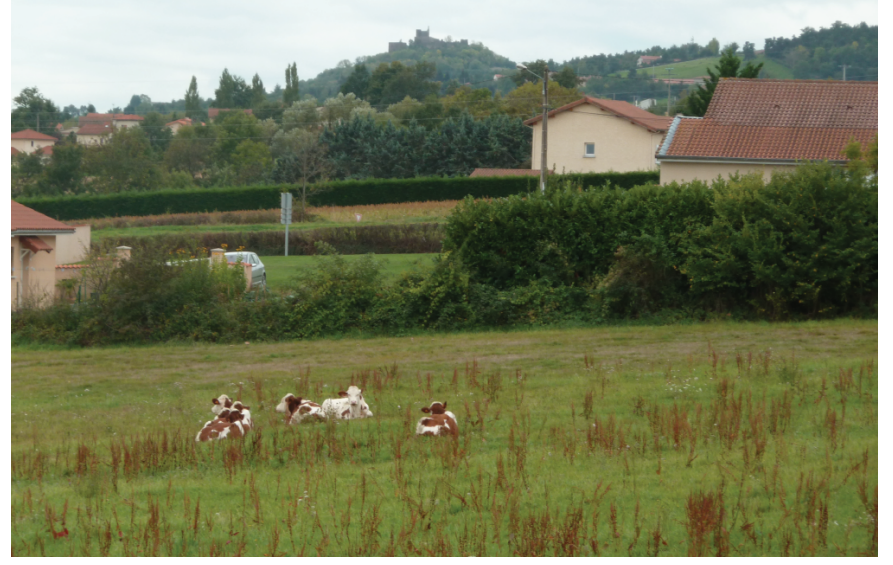

Photo 5. Distributeur d'œufs automatique dans le périurbain d'Amiens (Photo Adrien Baysse-Lainé).

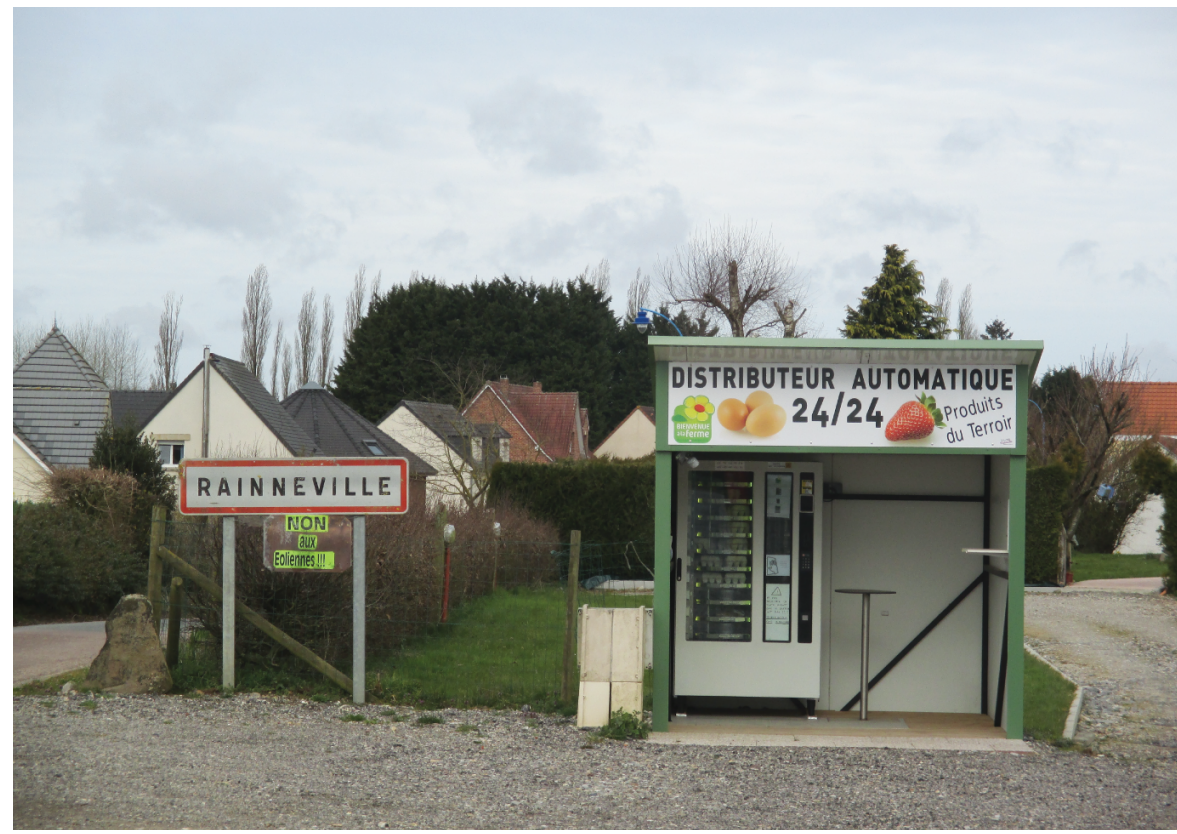

petits élevages à caractère alimentaire, parfois clandestins, près des villes de l'Est et des Balkans (Vallerand et al 2007) et réhabiliter les systèmes de semi-subsistance (par exemple en Pologne) au nom de la qualité des produits et des services environnementaux qu'ils fournissent (Smith et Jehlicka 2013).

\section{1 / Un service d'approvisionne- ment dynamisé par la vente directe et l'accueil à la ferme}

Un nombre croissant d'élevages profite de la proximité urbaine pour la commercialisation de leur production via la vente directe et l'accueil à la ferme. Les circuits courts offrent des possibilités d'installation y compris pour des systèmes conventionnels (Tredan 2016). Des élevages caprins s'installent près de villes sur de petites superficies. Bien qu'ils ne soient en général pas autonomes pour l'alimentation des animaux (Testard 2014), leurs produits ont une bonne image auprès des consommateurs et sont écoulés sur le marché urbain. Dans les territoires où les filières laitières sont historiquement organisées en circuits longs, de nouvelles opportunités s'offrent aux éleveurs pour valoriser le lait : glaces fermières, produits frais, souvent avec un accueil pédagogique (Le Gall 2013). Il en est de même pour la production de viande bovine (Duvernoy 2002, Vandenbrouck 2013). Les éleveurs de poules pondeuses innovent avec par exemple des distributeurs automatiques d'œufs (photo 5). Dans d'autres cas, l'élevage avicole périurbain approvisionne des métropoles plus éloignées, comme par exemple celui autour d'Amiens qui approvisionne à la fois Amiens et Paris. L'aviculture de loisir a aussi des débouchés alimentaires grâce à l'autoconsommation (ou la vente) des œufs et des animaux non conformes aux critères de sélection, ce qui permet de réduire son coût (Soulard et Thareau 2009). Enfin, les animaux d'élevage se maintiennent à la frange immédiate du tissu urbain grâce aux exploitations des lycées agricoles, qui dans certaines villes moyennes de régions d'élevage ont aussi un atelier de transformation et un magasin de vente (Arnal 2012).

\section{2 / De nombreux services envi- ronnementaux et paysagers en réponse au besoin de "nature " des citadins}

Les services environnementaux rendus par l'élevage périurbain sont soulignés dans les travaux traitant de la multifonctionnalité de l'agriculture (Zasada 2011). L'élevage d'herbivores permet de maintenir des espaces ouverts et répond aux attentes des habitants et des élus pour des paysages attractifs et des espaces récréatifs à proximité immédiate des villes (van Oudenhoven et al 2012). Ces espaces relativement préservés motivent souvent le choix d'installation de nouveaux habitants. En France, l'entretien des trames vertes et bleues amène à concevoir de nouveaux modes de gestion des espaces non bâtis en particulier par le pâturage. Les élevages d'herbivores qui utilisent ces espaces fournissent aussi des aménités paysagères (Alavoine Mornas et Girard 2014, Tredan 2016). Ils gèrent des espaces « à risque ", par exemple des vallées sur lesquelles pèse un risque d'inondation (Soulard et Thareau 2009) ou préviennent les risques d'incendies dans les espaces périurbains méditerranéens (photo 6). 
Photo 6. Élevage d'ânes dans le périurbain de la Côte d'Azur : les ânes restent en hiver pour " débroussailler les mimosas » et retournent en estive l'été dans les Hautes-Alpes où est situé le siège d'exploitation (C. Delfosse).
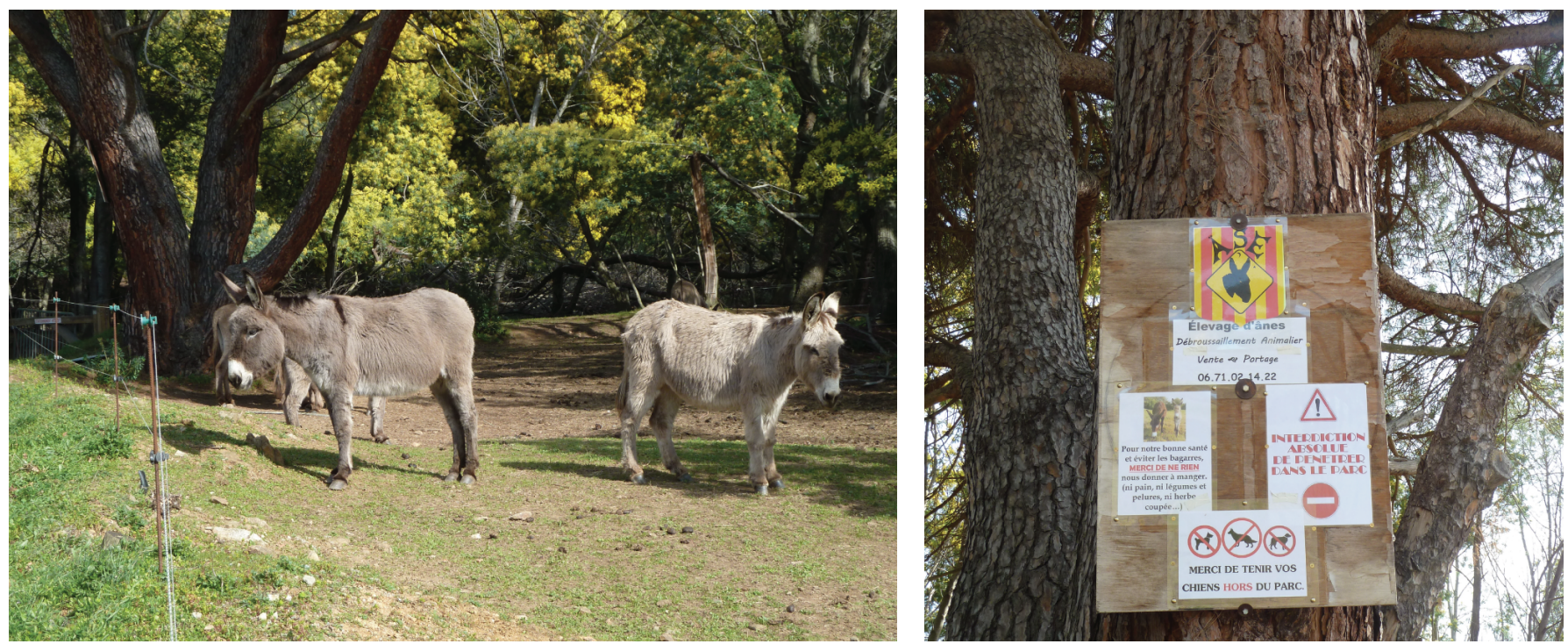

Photo 7. Jasseron, périurbain de Bourg-en-Bresse : cheval dans une propriété individuelle (à gauche) et élevage équestre (à droite, photos C. Delfosse).
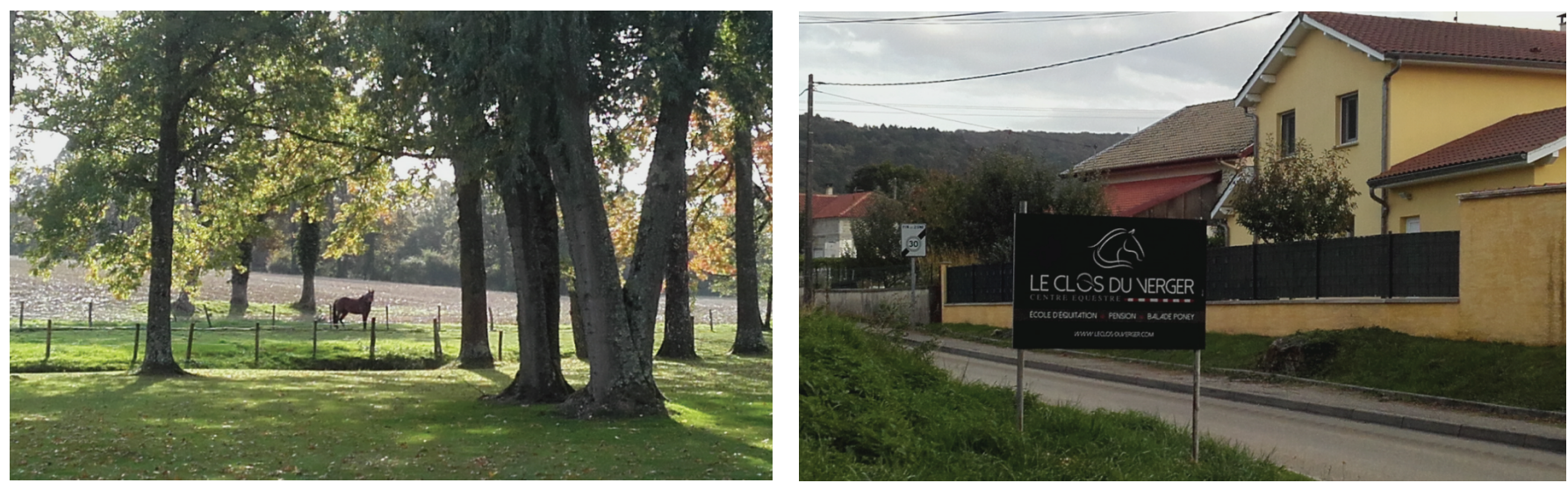

Dans des espaces à protéger autour de Stockholm, les éleveurs perçoivent des indemnités en échange de pratiques non rentables afin de satisfaire aux objectifs paysagers recherchés (Hochedez 2014b). Des exemples semblables existent aux Pays-Bas (Pfeifer 2011). Près de Grenoble, un élevage de chèvres et une fromagerie intercommunale maintiennent un espace de respiration indispensable aux grenoblois, tout en alimentant des circuits courts et en étant le support d'un projet pédagogique (Vargas 2013). De telles combinaisons se retrouvent dans les parcs agricoles périurbains italiens et espagnols (Marraccini et al 2013, Serrano 2015). Certes ceux-ci sont principalement voués à l'agriculture maraîchère mais ils promeuvent aussi des actions en faveur de l'élevage (label pour les poulets élevés dans le parc de Llogat à Barcelone, balades à cheval).

Dans le cadre du Grenelle de l'environnement, les projets en faveur de l'offre de produits locaux dans les cantines offrent aussi des débouchés aux élevages périurbains et sensibilisent les collectivités à l'agriculture (Praly et al 2012).
Toutefois, les politiques publiques en faveur de l'environnement, notamment l'élaboration de périmètres de protection agricole, peuvent fragiliser l'élevage périurbain.

Les produits issus des animaux élevés dans les espaces périurbains et destinés à l'alimentation locale réduisent aussi la consommation de fuel pour le transport et l'emballage (Voigt 2011). L'élevage avicole peut valoriser les résidus des cultures légumières. Ainsi dans les environs de Stockholm les exploitations biologiques de petite taille évoluent vers des systèmes de polyculture-élevage afin de réduire leurs coûts de production. Une exploitation avicole contribue par exemple à la fertilisation de pieds de tomates, et des oies désherbent des fraisiers (Hochedez 2014a). Le fumier de cheval est un engrais apprécié par les jardiniers et les maraîchers. Enfin, les contrats entre les communes et les exploitations d'élevage voisines permettent de valoriser les effluents d'élevage pour produire de l'énergie (Dourmad et al 2017, ce numéro). Celle-ci est souvent utilisée à courte distance ; le poids démographique des villes incite donc à la localisation de projets de méthanisation dans les espaces périurbains (Tritz 2013).

\section{3 / Des services sociaux et de vitalité territoriale très spécifiques}

Les nouvelles formes que prend l'élevage périurbain peuvent être le fait d'agriculteurs à temps partiel ou faire l'objet de créations d'entreprises par des nonagriculteurs. Les élevages de loisirs donnent lieu à de véritables PME (Soulard et Thareau 2009). Les activités équestres, particulièrement développées autour des villes, sont en particulier intégrées à différents circuits commerciaux et techniques : vente des animaux à des négociants, contrats avec des vétérinaires, demande de prestation de service aux agriculteurs voisins (Alavoine-Mornas et Girard 2014). Elles permettent aux agriculteurs de vendre ou de louer aux centres équestres et aux propriétaires de chevaux des petites parcelles peu rentables à exploiter (Quetier et Gordon 2003 ; photo 7). Les centres équestres se concentrent le long des grands axes de communication (Vial et al 2011). Les fermes 
pédagogiques (Lescureux 2003), voire des fermes d'animation qui interviennent dans les villes mais ont leur siège dans le périurbain proche, profitent également de petites surfaces disponibles dans les espaces périurbains. Enfin, les fermes du care ou du « social farming » qui se développent beaucoup au Royaume-Uni et aux Pays-Bas (Hassink et al 2012, Leck et al 2014) possèdent en général des animaux d'élevage (Dumont et al 2016).

Des élevages de chiens, de gibier et d'oiseaux exotiques (Jarrige 2004) se développent également dans les espaces périurbains car ils n'ont besoin que d'un foncier restreint ; ils trouvent des débouchés immédiats auprès des citadins ou des sociétés de chasse. Ainsi, dans le périurbain lillois, des exploitations laitières se sont reconverties dans l'élevage de chiens et dans l'activité de pension suite au grignotage de leurs terres (Rouget 2013).

Le recul de l'élevage entraine aussi le recul des infrastructures (abattoirs, unités de transformation) qui lui sont liées, obérant parfois le devenir d'autres exploitations (Poulot et Rouyres 2004). Le manque d'infrastructures, notamment pour l'abattage des animaux, limite les installations d'élevage en circuit-court. À l'inverse, certaines industries agroalimentaires comme les anciennes fruitières de Savoie trouvent un nouvel élan en se transformant souvent en structure de vente, profitant de l'engouement pour les circuits courts. Là où les industries agroalimentaires et les abattoirs se sont maintenus, ils génèrent quelques emplois (Arnal 2012). Ces activités sont toutefois fragilisées par les difficultés de circulation des camions d'approvisionnement, les nuisances occasionnées aux riverains, et leur réticence à être à proximité immédiate des lieux de mise à mort des animaux.

\section{4 / Des freins principalement liés à la pression foncière et aux nuisances occasionnées par les animaux}

Un enjeu fondamental pour le maintien et le développement des élevages périurbains tient aux compromis et aux conflits liés au foncier (Bertrand 2013, Perrin 2017). La concurrence foncière accentue les tensions entre différents types d'élevage, ainsi qu'entre agriculteurs et éleveurs (Delfosse et al 2008). Paradoxalement la précarité périurbaine profite à des élevages ruraux dont les animaux peuvent utiliser l'herbe et les friches à proximité immédiate des villes. On assiste alors à une forme de « transhumance inversée » lorsqu'en hiver ces éleveurs font pâturer leurs animaux dans des espaces périurbains (Soulard 2014).
Les exploitations installées dans le périurbain proche font toutefois face à une forte pression foncière qui réduit le nombre de parcelles destinées au pâturage et conduit à un émiettement du parcellaire. La circulation automobile pèse sur les exploitations herbagères lorsque les éleveurs changent leurs animaux de parcelles. Cela peut les conduire à intensifier leur système de production, ce qui est préjudiciable pour les exploitations en AOP comme l'ont analysé Delattre et al (2005) dans le cas de la Savoie. Toutefois, même si la proximité urbaine fragilise les exploitations AOP dans les espaces périurbains, elle leur offre aussi d'importants débouchés auprès des consommateurs locaux. Ceci a par exemple dynamisé des AOP telles que le bleu de Gex près de Genève, ou les bries de Meaux et de Melun en Ile-deFrance (Delfosse 2015).

La fragilité de l'élevage dans ces espaces tient également aux nuisances occasionnées par les animaux (bruits, odeurs, poussière, présence d'insectes et de rongeurs attirés par les élevages) ainsi que par l'évolution des normes sanitaires et environnementales. Cela concerne surtout les élevages avicoles (Henderson 2003) et porcins (Dourmad et al 2017), mais n'épargne pas l'élevage laitier (Sneeringer 2011) ni les centres équestres (Vial et al 2011). À proximité des agglomérations de l'arc alpin, le nombre de conflits entraînant la délocalisation des bâtiments d'élevage se sont ainsi multipliés (Delattre et al 2005); dans les Alpes du Nord, les effectifs bovins ont chuté de $25 \%$ dans les communes urbaines entre 1988 et 2000 , alors que la baisse des effectifs totaux n'était que de $6 \%$. La construction de nouveaux bâtiments et l'agrandissement des bâtiments existant

Figure 2. Représentation synthétique du bouquet de service issu de l'élevage périurbain.

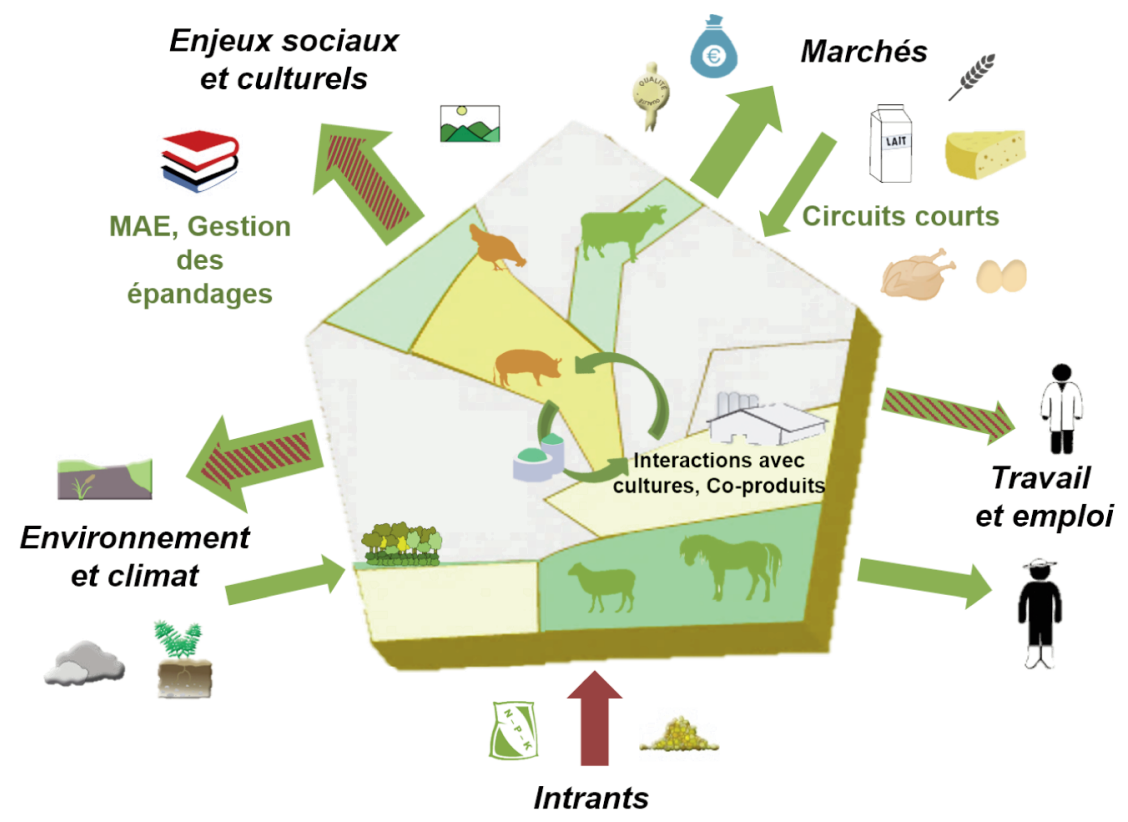
des habitants contre les permis de construire, et par des contraintes d'urbanisme propres aux espaces périurbains (Nougarèdes 2013).

\section{5 / Au final, un bouquet de services large et assez équilibré}

L'élevage offre une grande diversité de services dans les espaces périurbains, comme l'ont également souligné les travaux de chercheurs néerlandais (van Oudenhoven et al 2012) et danois (Turner et al 2014). Les exploitations d'élevage contribuent au service d'approvisionnement, et la commercialisation des produits profite de l'engouement d'une partie de la population pour les circuits courts. Ces exploitations subissent une forte pression foncière, mais présentent des atouts pour le bien-être des citadins qui aiment voir des animaux à proximité de chez eux. Au-delà des services culturels (zones récréatives, patrimoine naturel et bâti), l'élevage d'herbivores permet de maintenir des prairies qui fournissent différents services de régulation (stockage du carbone dans le sol, captage des particules fines, limitation de l'impact des crues). Les principaux services et impacts des systèmes d'élevage périurbains sont synthétisés dans la figure 2 et le tableau 2.

\section{Conclusion}

Notre synthèse rassemble une littérature peu nombreuse et encore fragmentaire sur les rôles, impacts et services de l'élevage dans les espaces urbains et périurbains en Europe. Parmi les points de convergence, le lien que souhaite peuvent être contrariés par des recours 
Tableau 2. Atouts et impacts associés à l'élevage périurbain.

\begin{tabular}{|c|c|c|}
\hline Interfaces étudiées & Effets positifs & Effets négatifs \\
\hline Intrants & $\begin{array}{l}\text { - Moindre consommation d'Energie du fait } \\
\text { des distances réduites (systèmes } \\
\text { alimentaires locaux) } \\
\text { - Contribution à la production d'Energie }\end{array}$ & $\begin{array}{l}\text { - Effets délocalisés pour la production } \\
\text { d'aliments concentrés } \\
\text { - Fertilisation minérale des prairies, pesticides } \\
\text { utilisés sur cultures }\end{array}$ \\
\hline Travail et emploi & $\begin{array}{l}\text { - Quelques emplois directs en élevage } \\
\text { (+ vente directe) } \\
\text { - Emploi indirect : pensions, etc }\end{array}$ & $\begin{array}{l}\text { - Recul infrastructures de services : abattoirs, } \\
\text { transformation, vétérinaires, etc }\end{array}$ \\
\hline Marchés & $\begin{array}{l}\text { - Approvisionnement en produits frais (intérêt } \\
\text { accru par la crise économique) } \\
\text { - Produits avec potentiel de valeur ajoutée } \\
\text { (circuits courts) }\end{array}$ & \\
\hline $\begin{array}{l}\text { Enjeux sociaux } \\
\text { et culturels }\end{array}$ & $\begin{array}{l}\text { - Entretien d'espaces récréatifs et de la } \\
\text { trame paysagère } \\
\text { - Fermes pédagogiques } \\
\text { - Découverte de la fabrication des produits }\end{array}$ & $\begin{array}{l}\text { - Nuisances olfactives (déjection) et sonores } \\
\text { - Conflits liés à l'étalement urbain : gêne au } \\
\text { trafic routier... } \\
\text { - Perception visuelle des bâtiments d'élevage }\end{array}$ \\
\hline $\begin{array}{l}\text { Environnement } \\
\text { et climat }\end{array}$ & $\begin{array}{l}\text { - Entretien de l'espace : préservation contre } \\
\text { incendies, crues } \\
\text { - Biodiversité commune (prairies, haies) et } \\
\text { domestique (aviculture, chèvres) }\end{array}$ & $\begin{array}{l}\text { - Risques de pollutions des nappes } \\
\text { (évacuation des fumiers et lisiers) } \\
\text { - Intensification des systèmes en raison du prix } \\
\text { du foncier }\end{array}$ \\
\hline
\end{tabular}

retrouver une frange croissante des citadins avec les produits qu'ils consomment représente une opportunité majeure. L'élevage est ainsi directement en prise avec les débats autour de la gouvernance alimentaire des villes, ce qui lui est en général favorable (développement des circuits courts, recherche de produits de proximité) mais peut parfois s'avérer défavorable (vigilance accrue par rapport au bien-être animal). Ainsi, pour se développer l'élevage devra-t-il probablement se différentier des productions industrielles classiques. En Europe, le rôle d'approvisionnement qu'a l'élevage urbain est jusqu'ici mineur, même s'il semble plus important en Europe de l'Est et méditerranéenne. La question du foncier est fondamentale pour l'élevage périurbain : le coût des terres est défavorable à des pratiques extensives alors que l'élevage d'herbivores en périphérie des villes entretient des espaces à usage récréatif et préserve des prairies qui fournissent différents services de régulation. La question des nuisances occasionnées par les animaux se pose dans les deux types d'espaces mais de manière plus marquée dans le périurbain du fait des densités animales plus élevées.

La littérature scientifique ne permet pas à ce jour de quantifier l'importance du service d'approvisionnement rendu par l'élevage urbain et périurbain, et ses variations régionales, et ceci bien qu'il existe un nombre croissant d'études qui décrivent et analysent son rôle dans l'approvisionnement des villes (Chatzimpiros et Barles 2010, Groshens et al 2016, Baysse-Lainé et Perrin 2017).
Une meilleure quantification du service d'approvisionnement permettrait de mieux comprendre le développement de l'élevage urbain et périurbain et sa dynamique d'évolution dans ces deux types d'espaces. Une question concerne plus particulièrement la contribution de l'élevage urbain aux projets alimentaires territoriaux; le service d'approvisionnement n'y est pas central, du moins en Europe de l'Ouest, mais l'élevage peut contribuer à resserrer les liens entre les citadins et les modes de production de leur nourriture.

Un autre phénomène qui nécessitera d'être quantifié est le rôle de l'élevage urbain et périurbain pour réduire les gaspillages. Les déchets alimentaires urbains sont récupérés soit pour l'alimentation des animaux élevés dans ces espaces soit, en complément des déjections humaines et animales, comme amendement agricole pour restituer des nutriments aux sols des terres arables. Dans un scénario où les monogastriques valoriseraient les déchets alimentaires et de l'industrie agroalimentaire, et les coproduits des cultures, la production de porcs dans l'Union Européenne verrait ses besoins en surface réduits d'un cinquième (zu Ermgassen et al 2016). Des questions se posent toutefois sur les risques de propagation de maladies (Guilbert et al 2015), si bien que de telles évolutions dans la manière d'alimenter les animaux nécessiteraient de mettre en place une réglementation sanitaire stricte pour collecter les déchets, les stocker et les transformer (Dumont et al 2017, ce numéro).
Dans cette synthèse, nous avons évoqué que l'élevage générait différents types d'emplois dans les espaces urbains et périurbains. Le nombre et le type d'emplois potentiellement créés doivent toutefois être quantifiés. On constate que les métiers d'éleveurs seront divers dans ces espaces avec des particuliers qui possèdent quelques animaux mais n'ont pas un statut « éleveur », des éleveurs professionnels pour qui l'élevage procure une part plus ou moins importante du revenu, des salariés de municipalités, des entreprises de gestion des espaces verts, etc. Cette diversité de statut mériterait d'être mieux identifiée et caractérisée car elle questionne l'évolution des activités et des formes de travail propres à l'agriculture.

Enfin, se pose la question du degré d'organisation ou de précarité de ces formes d'élevage. Les premiers éléments issus de la littérature permettent de faire l'hypothèse de spécificités en Europe de l'Est (Guest 2003, Smith et Jehlicka 2013), méditerranéenne (Jouve et Padilla 2007, Perucho et al 2015) et dans les Balkans (Vallerand et al 2007). Dans le cas de l'élevage périurbain, on pourra questionner si l'importance de ces exploitations pour le bien-être des citadins pourra à terme leur assurer des aides des collectivités territoriales allégeant la pression foncière. Dans le cas de l'élevage urbain, il semble que les expériences, souvent ponctuelles, ne pourront se développer qu'avec le soutien des villes (pour le foncier, les débouchés et la maind'œuvre), des consommateurs, voire de l'État dans le cas des jardins d'insertion. 


\section{Références}

Alavoine Mornas F., Girard S., 2014. Foncier agricole et enjeux environnementaux : de nouvelles tensions au niveau des exploitations agricoles pour la mise en oeuvre de la Trame Verte. Sci. Eaux Terr., 19, 46-49.

Arnal C., 2012. La place et le rôle de l'agriculture à la périphérie des villes moyennes. Le cas des villes d'Annecy, Bourg-en-Bresse, Montbrison et Romans-sur-Isère. Thèse de Géographie, Université Lumière, Lyon 2, France.

Ba A., Aubry C., 2011, Diversité et durabilité de l'agriculture urbaine : une nécessaire adaptation des concepts? Norois, 221, 11-24.

Baratay E., 2011, Bêtes de somme - Des animaux au service des hommes. Editions Seuil « Points Histoire », Paris, France, 442, 144p.

Barreau D., 2014, Agir pour l'agriculture : Nantes Métropole, Pour, 224, 351-360.

Baysse-Lainé A., Perrin C., 2017. Les espaces agricoles des circuits de proximité : une lecture critique de la relocalisation de l'approvisionnement alimentaire de Millau. Nat. Sci. Soc., 25 , 21-35.

Bertrand N., 2013. Terres agricoles périurbaines. Une gouvernance foncière en construction. Éditions Quae, Versailles, France, 250p.

Blanc N., 2000. Les Animaux et la Ville, Odile Jacob, Paris, France, 232p.

Bonnefoy S., Brand C., 2014. Régulation politique et territorialisation du fait alimentaire : de l'agriculture à l'agri-alimentaire. Géocarrefour, 89, 95-103.

Bories O., Eychenne C., 2016. L'éco pâturage : une nouvelle pratique qui interroge l'espace public. Renc. Rech. Rum., 23, 177-180

Chatzimpiros P., Barles S., 2010. Nitrogen, land and water inputs in changing cattle farming systems. An historical comparison for France, $19^{\text {th }}-21^{\text {st }}$ centuries. Sci. Total Environ., 408, 4644-4653.

Chemla G., 1994. Les Ventres de Paris, les Halles, la Villette, Rungis : l'histoire du plus grand marché du monde. Éditions Glénat, Grenoble, France, 210p.

Csergo J., Lemasson J.P., 2008. Voyages en gastronomies. L'invention des capitales et des régions gourmandes. Éditions Autrement, Paris, France, 264p.

Darly S., 2014. Des moutons dans la ville : quelles externalités environnementales des pratiques d'élevage ovin en milieu urbain ? Pour, 224, 285-290.

Darribehaude F., Gardon S. Lensel B., 2016, Le vivant en ville, Nouvelles émergences : gouvernance, intensité urbaine, agriculture et animalité urbaines. Éditions Grand Lyon, la Métropole, 224p.

Delattre F., Hauwuy A., Perron L., 2005. The AOC label for Savoyard cheeses : past dynamics, achievements and challenges in the today's changing context (CAP, urban development). J. Alp. Res., 4, 119-126.

Delfosse C., 2001. Les multiples facettes des cultures territoriales dans le département du Nord. Hommes et terres du Nord, 4, 205-213.
Delfosse C., 2011. La patrimonialisation des produits dits de terroir : l'occasion d'une confrontation entre agriculteurs et citadins/nouveaux ruraux. Anthr. Food, 8, 153-166.

Delfosse C., 2014. Le métier de crémier-fromager de 1850 à nos jours. Éditions de la mer du Nord, Boulogne, 176p.

Delfosse C., 2015. L'intégration à l'INAO d'un autre secteur AOC développé : les produits laitiers. In: Une histoire des vins et des produits AOC. L'INAO de 1935 à nos jours, Wolikow S., Humbert F. (Éds). Presses universitaires de Dijon, France, 161-180.

Delfosse C., Sainte-Marie C., Perenzin C. 2008, Multi-usage des territoires ruraux et agriculture. Les éleveurs caprins dans le Parc naturel régional des Bauges. In : Nouvelles questions agraires. Exploitants, fonction et territoires. Mayaud J.L., Cornu P. (Éds). La Bibliothèque de l'Histoire, Paris, France, 157-182.

Dourmad J.Y., Delaby L., Boixadera J., Ortis C., Méda M., Gaigné C., Dumont B., 2017. Diversité des services rendus par les territoires à forte densité d'élevage, trois cas d'étude en Europe. In : Numéro spécial, L'élevage en Europe : une diversté de services et d'impacts. Dumont B. (Éd). INRA Prod. Anim., 30, 303-320.

Dumont B., Fortun-Lamothe L., Jouven M. Thomas M., Tichit M., 2013. Prospects from agroecology and industrial ecology for anima production in the $21^{\text {st }}$ century. Animal, 7, 1028 1043.

Dumont B. (coord.), Dupraz P. (coord.), Aubin J., Batka M., Beldame D., Boixadera J., BousquetMelou A., Benoit M., Bouamra-Mechemache Z., Chatellier V., Corson M., Delaby L., Delfosse C., Donnars C., Dourmad J.Y., Duru M., Edouard N., Fourat E., Frappier L., FriantPerrot M., Gaigné C., Girard A., Guichet J.L., Haddad N., Havlik P., Hercule J., Hostiou N., Huguenin-Elie O., Klumpp K., Langlais A., Lemauviel-Lavenant S., Le Perchec S., Lepiller O., Letort E., Levert F., Martin, B., Méda B. Mognard E.L., Mougin C., Ortiz C., Piet L., Pineau T., Ryschawy J., Sabatier R., Turolla S. Veissier I., Verrier E., Vollet D., van der Werf H., Wilfart A., 2016, Rôles, impacts et services issus des élevages en Europe. Rapport de l'Expertise scientifique collective réalisée par l'INRA à la demande des ministères en charge de l'Écologie et de l'Agriculture, et de l'Ademe, INRA, Paris, France, 1032p.

Dumont B., Ryschawy J., Duru M., Benoit M., Delaby L., Dourmad J.Y., Méda B., Vollet D., Sabatier R., 2017. Les bouquets de services, un concept clé pour raisonner l'avenir des territoires d'élevage. In : Numéro spécial, L'élevage en Europe : une diversité de services et d'impacts. Dumont B. (Éd). INRA Prod. Anim., 30, 407 422.

Duru M., Donnars C., Ryschawy J., Therond O., Dumont B., 2017. La "grange": un cadre conceptuel pour appréhender les bouquets de services rendus par l'élevage dans les territoires. In : Numéro spécial, L'élevage en Europe une diversité de services et d'impacts. Dumont B. (Éd). INRA Prod. Anim., 30, 273-284.

Duvernoy I., 2002. Vente directe de viande bovine dans la vallée du Thoré (Tarn) : un exemple de diversification agricole en proximité urbaine. Cah. Agric., 11, 137-143.
Etard A., 2014. Abeilles maraîchères, un jardin d'insertion par l'activité économique francilien. Pour, 224, 215-224.

Groshens E., Dockès A.C., Drouin R., Lesty M., 2016. Construire les futurs de l'élevage en Île-de-France, Renc. Rech. Rum., 23, 173-176.

Guest M., 2003. La ruralité des capitales balkaniques. L'exemple de Sofia. Balkanologie, 7 , $127-150$.

Guilbert S., Redlingshöfer B., Gracieux M., Fuentes C., 2015. Systèmes alimentaires urbains : comment réduire les pertes et gaspillages alimentaires? Rapport d'étude, INRA, Paris, France, $45 \mathrm{p}$.

Hassink J., Hulsink W., Grin J., 2012. Care farms in the Netherlands: An underexplored example of multifunctional agriculture. Toward an empirically grounded, organization-theorybased typology. Rural Sociol., 77, 569-600.

Henderson S.R., 2003. Agricultural adaptation to real regulation on the urban fringe: the chicken meat industry's response to land-use conflict in the Western Port Region of Victoria, Australia. Austr. Geogr. Stud., 41, 156-170.

Hercule J., Chatellier V., Piet L., Dumont B., Benoit M, Delaby L., Donnars C., Savini I., Dupraz P., 2017. Une typologie pour représenter la diversité des territoires d'élevage en Europe. In : Numéro spécial, L'élevage en Europe : une diversité de services et d'impacts. Dumont B. (Éd). INRA Prod. Anim., 30, 285-302.

Hochedez C., 2014a. L'agriculture biologique : une agriculture au service de l'environnement local autour de Stockholm. Pour, 224, 275-283.

Hochedez C., 2014b. La mise en place des politiques alimentaires locales dans la région métropolitaine de Stockholm : une gouvernance du malentendu? Géocarrefour, 89, 115-124.

Huang D., Drescher M., 2015. Urban crops and livestock: The experiences, challenges, and opportunities of planning for urban agriculture in two Canadian provinces. Land Use Policy, $43,1-14$

Jarrige F., 2004. Les mutations d'une agriculture méditerranéenne face à la croissance urbaine dynamiques et enjeux autour de Montpellier. Cah. Agric., 13, 64-74.

Jouve A.M., Padilla M., 2007. Les agricultures périurbaines méditerranéennes à l'épreuve de la multifonctionnalité : comment fournir aux villes une nourriture et des paysages de qualité ? Cah. Agric., 16, 311-317.

Klinger D., Naylor R., 2012. Searching for solutions in aquaculture: charting a sustainable course. Annu. Rev. Environ. Resour., 37, $247-$ 276.

Kuiken T., Fouchier R., Rimmelzwaan G., van den Brand J., van Riel D., Osterhaus A., 2011. Pigs, poultry, and pandemic influenza: how zoonotic pathogens threaten human health. Adv. Exp. Med. Biol., 719, 59-66.

Labouesse F., 1998. La construction de nouvelles relations entre monde agricole et société : une approche à partir de fêtes de la transhumance. Ruralia, 02 | 1998 http://ruralia.revues.org/33 
Lardon L., Loudiyi S., 2014. Agriculture urbaine et alimentation : entre politiques publiques et initiatives locales. GeoCarrefour, 89, 3-10.

Leck C., Evans N., Upton D., 2014. AgricultureWho cares? An investigation of 'care farming'in the UK. J. Rural Stud., 34, 313-325.

Le Gall P., 2013. L'économie agricole dans l'aire urbaine d'Annecy, le cas de la filière lait et ses modes de vente. Mémoire de M1 (Géographie). Université Lyon 2, France,148p.

Lescureux F., 2003. Les relations des agriculteurs au territoire au travers de la vente directe et de l'accueil à la ferme : le cas de la région des Monts de Flandres. Doctorat de Géographie, Université de Lille I, France, 602p.

Love D.C., Fry J.P., Li X.M., Hill E.S., Genello L., Semmens K., Thompson R.E., 2015. Commercial aquaponics production and profitability: Findings from an international survey. Aquaculture, 435, 67-74.

Lovell S.T., 2010. Multifunctional urban agriculture for sustainable land use planning in the United States. Sustainability, 2, 2499-2522.

Marraccini E., Lardon S., Loudiyi S., Giacché G., Bonari E., 2013. Durabilité de l'agriculture dans les territoires périurbains méditerranéens : enjeux et projets agriurbains dans la région de Pise (Toscane, Italie). Cah. Agric., 6, 517-525.

Maudet J.B., 2006. Le taureau marque son territoire (festivités taurines et identités territoriales du Sud-ouest européen à l'Amérique Latine). Ann. Geogr., 650, 361-387.

McClintock N., Pallana E., Wooten H., 2014. Urban livestock ownership, management, and regulation in the United States: An exploratory survey and research agenda. Land Use Policy, $38,426-440$.

McShane C., Tarr J.A., 2007. The Horse in the City - Living Machines in the Nineteenth Century. Johns Hopkins University Press, Baltimore, Maryland, 242p.

Milan B.F., Creutzig, F., 2015. Reducing urban heat wave risk in the $21^{\text {st }}$ century. Curr. Opin. Environ. Sustain., 14, 221-231.

Morgan K., 2009, Feeding the City: The Challenge of Urban Food Planning. Int. Plan. Stud., 14, 341-348.

Mundler P., Consalès J.N., Melin G., Pouvesle C., Vandenbroucke P., 2014. Tous agriculteurs ? L'agriculture urbaine et ses frontières. GeoCarrefour, 89, 53-63.

Nahmias P., Le Caro, Y., 2012, Pour une définition de l'agriculture urbaine : réciprocité fonctionnelle et diversité des formes spatiales, EUE, 6, http://eue.revues.org/437

Nougarèdes, B., 2013. Modes d'insertion socio-spatiale du bâti agricole périurbain et sociabilités locales. Le cas des hameaux agricoles dans l'Hérault. Thèse de géographie Université de Toulouse.

Perrin C., 2017, Mobiliser la propriété publique en faveur de l'agriculture périurbaine? Le cas de la région de Montpellier, France. VertigO, 17 Numéro 1 | mai 2017, http://vertigo.revues. org/18401

Perrin C., Soulard C.T., 2014. Vers une gouvernance alimentaire locale reliant ville et agricul- ture. Le cas de Perpignan. GeoCarrefour, 89, 124-135.

Perrin C., Soulard C.T., 2017. Introduction. L'agriculture dans le système alimentaire urbain : continuités et innovations. Nat. Sci. Soc., 25, 3-6.

Perucho L., Bazin G., Goussios D., 2015. Crise économique grecque et nouvelles dynamiques agraires : l'exemple de la Thessalie orientale. Ann. Geogr., 705, 473-497.

Pfeifer C., 2011, Le rôle de l'agriculture pour soutenir la multifonctionnalité des territoires ruraux aux Pays-Bas. Urbia, 12, 67-83.

Poulot M., Rouyres T., 2004. L'Île-de-France agroalimentaire : vers de nouvelles relations territoriales entre IAA et agriculture ? In: Dynamiques agro-industrielles et dynamiques rurales. Margetic C. (Éd), Presses Universitaires d'Artois, Arras, 109-131.

Praly C., Chazoule C., Delfosse C., Saleilles S., Miehé A., 2012. Repenser l'échelle d'approvisionnement des cantines. In : Au plus près de l'assiette : pérenniser les circuits courts alimentaires. Prigent-Simonin A.H., Hérault-Fournier C. (Éds), Quae, Versailles, 169-186.

Quetier F.F., Gordon I.J., 2003. 'Horsiculture': how important a land use change in Scotland? Scot. Geogr. J., 119, 153-158.

Raudsepp-Hearne C., Peterson G.D., Bennett E.M., 2010. Ecosystem service bundles for analyzing tradeoffs in diverse landscapes. Proc. Natl. Acad. Sci., 107, 5242-5247.

Rouget N., 2013. Trajectoires et stratégies agricoles dans les espaces urbains et périurbains. Entre spécialisation et conversion. L'exemple de la périphérie Sud-Est de l'agglomération lilloise. Bull. Assoc. Geogr. Fr., 90, 286-302.

Serrano J.A.S., 2015. Agricultura periurbana, parques naturales agrarios y mercados agropecuarios locales: una respuesta territorial y productiva a la subordinación del campo a la ciudad. Scripta Nova., 19, http://www.ub.es/ geocrit/sn/sn-502.pdf

Smith J., Jehlicka P., 2013. Quiet sustainability: Fertile lessons from Europe's productive gardeners. J. Rural Stud., 32, 148-157.

Sneeringer, S.E., 2011. Effects of environmental regulation and urban encroachment on California's dairy structure. J. Agric. Resour. Econ., 36, 590-614.

Schneider L., 2014. La Ferme du Bonheur, une réponse au contexte urbain et social de Nanterre. Pour, 224, 247-254.

Soulard C.T., 2014. Les agricultures nomades, une caractéristique du périurbain. Pour, 4, 151-158.

Soulard C.T., Thareau B., 2009. Les exploitations agricoles périurbaines : diversité et logiques de développement. Innov. Agron., 5, 27-40.

Testard P., 2014. L'élevage caprin en Isère étude géographique d'une production fermière polymorphe à forte valeur culturelle. Mémoire de Master 2, Université de Lyon 2, France, 165p.

Torre A., Pham V.H., Simon, A., 2015. The ex-ante impact of conflict over infrastructure settings on residential property values: The case of Paris's suburban zones. Urban Stud., 52, 24042424.
Tredan R., 2016, Politiques d'aménagement des terres agricoles en périurbain, formes d'agriculture et enjeux de justice : analyse de deux innovations foncières instituées dans les PyrénéesOrientales, Mémoire de master 2, Université Lyon 2, France.

Tribol R.M., 2017. Urban pastoralism as environmental tool for sustainable urbanism in Romania and Eastern Europe. Procedia Environ. Sci., available online at www.sciencedirect.com

Tritz Y., 2013. Développement territorial et valorisation en circuit court des ressources énergétiques locales. Vers des systèmes énergétiques agri-territoriaux ? Thèse de Géographie, Université Lyon 2, France, 441p.

Turner K.G., Odgaard M.V., Bocher P.K., Dalgaard T., Svenning J.C., 2014. Bundling ecosystem services in Denmark: Trade-offs and synergies in a cultural landscape. Landsc. Urban Plan., 125, 89-104.

Vallerand F., Dubeuf J.P., Tsiboukas K., 2007. Le lait de brebis et de chèvre en Méditerranée et dans les Balkans : diversité des situations locales et des perspectives sectorielles. Cah. Agric., 16, 258-264.

Vandenbrouck P., 2013. Transformation de l'unité de production agricole, d'une exploitation sectorielle à une exploitation agricole territoriale. Exploitations agricoles, agriculteurs et territoires dans les Monts du Lyonnais et en Flandre intérieure de 1970 à 2010. Thèse de Géographie, Université Lyon 2, France, 607p.

van Oudenhoven A., Petz K., Alkemade R., Hein L., de Groot R., 2012. Framework for systematic indicator selection to assess effects of land management on ecosystem services. Ecol. Indic., 21, 110-122.

Vanier M., 2011, « La périurbanisation comme projet », Métropolitiques, 23 février 2011. https://www.metropolitiques.eu/La-periurbanisation-comme-projet.html.

Vargas L., 2013. La ferme intercommunale des Maquis. Pour, 220, 163-171.

Vial C., Perrier-Cornet P., Soulard C., 2011. Le développement des équidés de loisir en France : quels impacts sur les espaces ruraux et periurbains ? Fourrages, 207, 165-172.

Vial C., Wanneroy A., Le Velly R., 2015. Quand le cheval contribue au dynamisme des territoires : analyse de projets locaux innovants entrepris par des collectivités françaises. Monde du Tourisme, hors-série Juin 2015, 161-171.

Voigt K.A., 2011. Pigs in the backyard or the barnyard: removing zoning impediments to urban agriculture. B.C. Envtl Aff. L. Rev., 38, 537-566.

Ward J.D., Ward P.J., Mantzioris E., Saint C., 2014. Optimising diet decisions and urban agriculture using linear programming. Food Secur., 6, 701-718.

Zasada I., 2011. Multifunctional peri-urban agriculture - A review of societal demands and the provision of goods and services by farming. Land Use Policy, 28, 639-648.

zu Ermgassen E.K.H.J., Phalan B., Green R.E., Balmford A., 2016. Reducing the land use of EU pork production: where there's swill, there's a way. Food Policy, 58, 35-48. 


\title{
Résumé
}

L'élevage dans les espaces urbains et périurbains trouve actuellement un net regain d'intérêt en Europe du fait de l'intérêt croissant des consommateurs autour du « produire local » et du besoin de « nature " des citadins. Notre objectif est ici de synthétiser une littérature souvent fragmentaire et de structurer cette analyse grâce au cadre des bouquets de services. Pour les espaces périurbains, la littérature scientifique traite en priorité des enjeux d'aménagement pour répondre à l'approvisionnement alimentaire des villes. L'élevage en ville est bien moins étudié, ce qui nous a conduits à utiliser quelques travaux hors Europe et à consulter les sites internet de communautés urbaines et des sites associatifs. Les bouquets de services rendus par l'élevage et les freins à son développement sont très différents entre ces deux types d'espaces. Dans les villes d'Europe de l'Ouest le service d'approvisionnement reste minoritaire. La réintroduction des animaux en ville est largement motivée par une volonté de créer du lien social et constitue un facteur de réinsertion et d'éducation des citadins vis-à-vis de l'origine des produits qu'ils consomment. Les principaux freins sont liés aux nuisances et aux risques épidémiologiques occasionnés par les animaux. Dans les espaces périurbains, la contribution de l'élevage au service d'approvisionnement est plus importante et dynamisée par la vente directe. L'élevage d'herbivores maintient des prairies qui fournissent différents services de régulation et répondent aux attentes des citadins pour des espaces récréatifs à proximité immédiate des villes. Le développement de l'élevage est principalement contraint par la forte pression foncière qui s'exerce sur ces espaces. Nous concluons en proposant quelques pistes de recherches.

\begin{abstract}
Roles, impacts and services provided by livestock in European urban and peri-urban areas

Livestock farming in urban and peri-urban areas finds a renewed interest in Europe as it meets consumer expectations for local products and the need for nature of city-dwellers. We aimed to review a fragmentary literature and to structure this analysis using the bundles of services framework. For peri-urban areas, the literature focusses on urban food planning in order to satisfy city food supply. Livestock farming in the urban areas is much less analysed, which led us to use some work outside Europe and to consult the websites of urban communities and some associative sites. Bundles of services provided by livestock farming are very different between these two types of areas. In Western European towns, provisioning services provided by livestock are minor compared with vegetables from urban gardens. Livestock animals mainly provide social services such as social reintegration, leisure and food education. Main limits are related to the odour, noise and epidemiological risks caused by the animals. Livestock farming systems are more productive in peri-urban areas where they benefit from consumer trust in local food systems. Herbivore farming provides various regulating services (climate, water and air quality, prevention of running fires) and meets the leisure expectations of city-dwellers: nature appreciation, horse riding centres, etc. Livestock farm development is mainly constrained by the high cost of land around cities. We conclude this review by proposing some research issues.
\end{abstract}

DELFOSSE C., DUMONT B., HOSTIOU N., 2017. Des services contrastés rendus par l'élevage dans les espaces urbains et périurbains européens. In : Numéro spécial, L'élevage en Europe : une diversité de services et d'impacts. Dumont B. (Éd). INRA Prod. Anim., 30, 395-406. 